\title{
A Study on Superconducting Coils for Superconducting Magnetic Energy Storage (SMES) Applications
}

\author{
Nuno Amaro ${ }^{1}$, João Murta Pina ${ }^{1}$, João Martins ${ }^{1}$, and José Maria Ceballos ${ }^{2}$ \\ ${ }^{1}$ Centre of Technology and Systems, Faculdade de Ciências e Tecnologia, \\ Universidade Nova de Lisboa, 2829-516 Caparica, Portugal \\ 2 "Benito Mahedero" Group of electrical Applications of Superconductors, \\ Escuela de Ingenierías Industriales, Universidad de Extremadura, Avenida de Elvas s/n, \\ 06006, Badajoz, Spain \\ nma19730@campus.fct.unl.pt, \{jmmp,jf.martins\}@fct.unl.pt, \\ jmceba@unex.es
}

\begin{abstract}
Superconducting coils (SC) are the core elements of Superconducting Magnetic Energy Storage (SMES) systems. It is thus fundamental to model and implement SC elements in a way that they assure the proper operation of the system, while complying with design specifications. As a part of a larger model, a coil design model is here presented and verified with tests made in a laboratory prototype. The limitations of the superconducting tape used, namely the negative effect of magnetic field components on its critical current value, are also verified and a possible solution to avoid that effect is studied.
\end{abstract}

Keywords: Superconducting Magnetic Energy Storage, SMES, High temperature superconductivity, HTS coil, HTS coil modeling.

\section{Introduction}

SMES system is a superconducting device with several possible applications in electric grids, and specially in the future's Smart Grids [1]. With such device it is possible to overcome several power quality issues, as voltage dips and swells, frequency oscillations or harmonic distortion [2]. Power quality is a very important topic in power grids, considering the huge dissemination of microprocessors and electronic devices. Electric energy is usually required to have high quality, i.e. with limited disturbances [3], making SMES a potential candidate in this field [1].

The operation principle of the SMES is based on storing energy in the magnetic field of a SC coil according to

$$
E=\frac{1}{2} L I^{2}
$$

where $E$ is the stored energy, $L$ is the inductance of the SC coil and $I$ is the current flowing in it. This energy is discharged into the grid when necessary. Since current 
flowing in the coil is DC and usually grids operate in AC, a bidirectional AC/DC converter and proper control strategies are necessary to assure energy exchanges with grid. This type of storage is only possible with superconducting materials, with virtually zero DC losses, as current decay due to e.g. copper resistivity would made it unfeasible.

The main goal of this work relies on deriving models for the SC coil that can be used in the design stage. These models must include not only electromagnetic effects but also mechanical and thermal characteristics of the coil. As a first approach, it was created a model to obtain the inductance of a coil given its geometric parameters. This model was then validated by comparing its results with measurements made in a real coil.

The coils used in experiments were implemented using first generation (1G) SC tape, built by $\mathrm{BiSrCaCuO}$ ceramic on its phase 2223. This material shows a significant drop in its critical current value in the presence of external magnetic field [4]. This degradation increases dramatically with components of magnetic field perpendicular to the tape. The influence of magnetic field in the value of the critical current is verified by measurements of critical current. In order to verify such dependence and improve the critical current a possible solution is also presented in the paper.

\section{Contribution to the Internet of Things}

Unlike existing unidirectional power grids, in electric grids of the future, energy is foreseen to flow up- and downstream. Smart Grids concept involves both energy and information flows on the grid [5]. To add information flow several standards and protocols must be created or adapted [6]. This paradigm change transforms the power grid in a completely new grid, much more similar to information networks like internet. In the last years several efforts are being made to create the concept of Internet of Things (IoT) in which every devices are connected to the Web. Smart Grids represent a particular case of IoT, where reliability and security of supply are requirements of utmost importance, considering energy transmission and distribution. Devices like SMES are foreseen to address these challenges, as well as contributing to integration of distributed generation, in a context of Smart Grids and IoT.

\section{$3 \quad$ Superconducting Coil Modeling}

\subsection{Geometry Based Model}

According to (1), stored energy in SMES depends on the current flowing through the $\mathrm{SC}$ coil and on its inductance. Since current depends on the superconducting material itself (critical current of the SC tape limits the admissible operation current) it is fundamental to maximize the inductance of the coil, so that the stored energy can be maximized while optimizing the required volume of material. Several approaches can 
be followed to determine the maximum inductance of a coil with specific geometric characteristics. In this case, a mathematical method was used. This method is based in classical formulas to determine self and mutual inductances of air core pancake coils $[7,8]$.

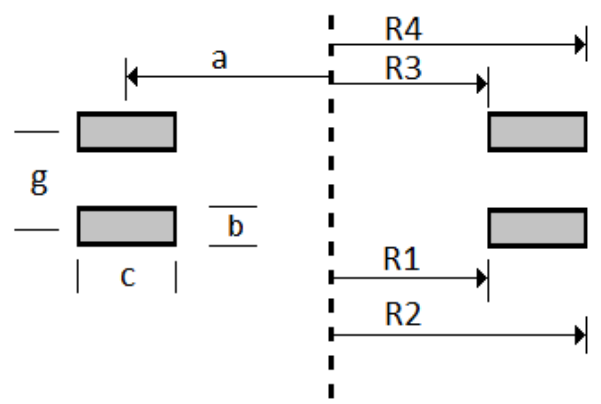

Fig. 1. Geometric variables of a set of air core pancake coils

Considering the geometric variables defined in Fig. 1, considering that $N$ is the number of turns of a single coil, then its self inductance $L$ in $\mathrm{mH}$, with dimensions in $\mathrm{cm}$, is approximated by

$$
L=4 \pi a N^{2}(\lambda+\mu),
$$

where parameters $\lambda, \mu$ and $x$ are defined by

$$
\begin{gathered}
\lambda=\log \left(\frac{8 a}{c}\right)+\frac{1}{12}-\frac{\pi x}{3}-\frac{\log \left(1+x^{2}\right)}{2}+\frac{\log \left(1+x^{2}\right)}{12 x^{2}}+ \\
+\frac{x^{2} \log \left(1+\frac{1}{x^{2}}\right)}{12}+\frac{2}{3}\left(x-\frac{1}{x}\right) \tan ^{-1}(x), \\
\mu=\frac{c^{2}}{96 a^{2}}\left\{\left[\log \left(\frac{8 a}{c}\right)-\frac{\log \left(1+x^{2}\right)}{2}\right]\left(1+3 x^{2}\right)+3.45 x^{2}+\frac{221}{60}-\right. \\
\left.-1.6 \pi x^{3}+3.2 x^{3} \tan ^{-1} x-\frac{\log \left(1+x^{2}\right)}{10 x^{2}}+\frac{x^{4} \log \left(1+\frac{1}{x^{2}}\right)}{2}\right\}, \\
x=b / c
\end{gathered}
$$

In order to compute mutual inductance $M$, also in $\mathrm{mH}$, between two coils with $N_{1}$ and $N_{2}$ turns, the following expression is used, with dimensions in $\mathrm{cm}$, 


$$
M=\frac{\mu_{0} N_{1} N_{2}}{\left(R_{2}-R_{1}\right)\left(R_{4}-R_{3}\right)} \int_{0}^{\pi} \int_{R_{1}}^{R_{2}} \int_{R_{3}}^{R_{4}} \frac{\cos (\theta) r_{1} r_{2}}{\sqrt{g^{2}+r_{1}^{2}+r_{2}^{2}-2 r_{1} r_{2} \cos (\theta)}} d r_{1} d r_{2} d \theta .
$$

If the SC coil is built only by one pancake, then inductance is given by (2)-(5). If more than one pancake coil is used, then mutual inductances between each pair of pancakes must be calculated and total inductance of the SC coil is given as follows

$$
L_{T}=L_{i}+2 \sum_{i=1}^{N_{C}-1} \sum_{j=2}^{N_{C}} M_{i j} \quad, i \neq j
$$

where $N_{C}$ is the number of pancakes in the set.

One common task consists on maximizing inductance of the coil given available length of superconducting tape. An optimization algorithm derived elsewhere [9] uses (2)-(5) to obtain internal radius and number of turns for a single coil that maximizes self inductance value. After this optimization, given the number of pancakes used, total inductance of the system is derived by (6)-(7).

\subsection{Validation of the Model}

To validate the results given by the model represented by equations (2)-(7), i.e. to verify that the values of mutual and self inductance given by equations are accurate, two small pancake coils were implemented using two similar 1G tapes (InnoST Bi-2223 HTS insulated wire), named A and B, whose characteristics are shown in Table 1.

Table 1. Characteristics of the SC tapes, according to supplier

\begin{tabular}{lcc}
\hline Characteristic & Tape A & Tape B \\
\hline Critical current @ 77 K, self-field (A) & 90 & \multicolumn{2}{c}{85} \\
Minimum bending radius (mm) & \multicolumn{2}{c}{30} \\
Width (mm) & \multicolumn{2}{c}{4.2} \\
Thickness (mm) & 0.25 \\
Available length for each coil (m) & \multicolumn{2}{c}{37.5} \\
\end{tabular}

The specified length of tape for each coil was $37.5 \mathrm{~m}$, and the optimization algorithm returned an internal radius of $32 \mathrm{~mm}$. After building coils with required dimensions, their inductance was measured using a digital RLC meter (LTD edc1620) and the results of such measurements are presented in Table 2. The implemented coils are shown in Fig. 2. As can be seen in Table 2, the results obtained with measurements are within a $10 \%$ margin of the calculated value, except for Coil A. Such deviation is due either to uncertainty in dimensions of the prototype due to shrinking of materials in liquid nitrogen $(77 \mathrm{~K})$ experimental measurements environment. 
Table 2. Comparison between model outputs and experimental measurements

\begin{tabular}{llll}
\hline & & Characteristic & Value $(\mathrm{mH})$ \\
\hline \multirow{4}{*}{ Model outputs } & Coil A & Self inductance & 1.75 \\
& Coil B & Self inductance & 1.75 \\
& Set & Mutual inductance & 2.5 \\
& & Total inductance & $\mathbf{6}$ \\
\hline \multirow{5}{*}{ Experimental measurements } & Coil A & Self inductance & 1.45 \\
& Coil B & Self inductance & 1.8 \\
& \multirow{2}{*}{ Set } & Mutual inductance & 2.7 \\
& & Total inductance & $\mathbf{5 . 9 5}$ \\
\hline
\end{tabular}

\section{Critical Current of the Superconducting Set of Coils}

Critical current measurement is needed as operating currents (and stored energy) are limited by this value. Manufacturers specify this value measured in a single portion of tape under self- or external field. In the present case, the effect of magnetic field due to adjacent stacked tapes is expected to degrade critical current. This value is often defined as the current that generates an electric field of $1 \mu \mathrm{V} / \mathrm{cm}$ in the tape.

\subsection{Critical Current Measurement}

Using the four point method, critical current of the two pancakes operating both separated and in series was measured and results are presented in Fig. 3. In both cases voltage drops at the terminals of each pancake were measured and added to obtain the total voltage drops in the SC set. Using this method, the voltage drop in the resistive connection between the two pancake coils does not influence results.

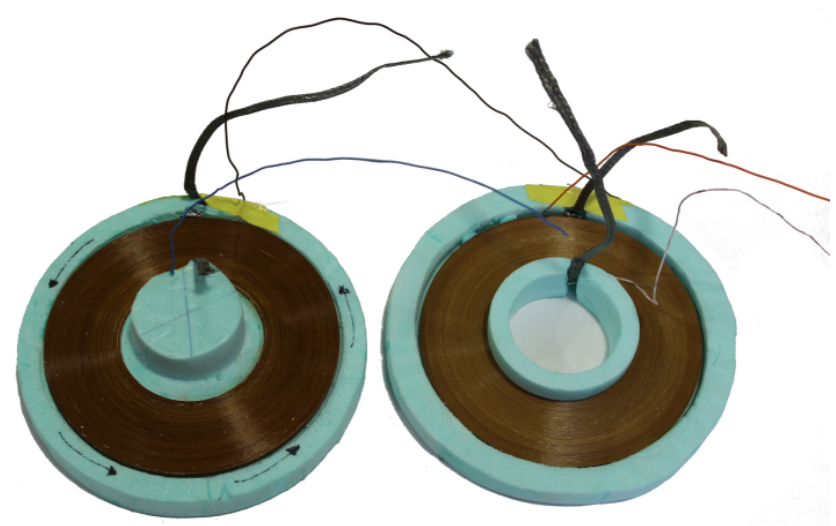

Fig. 2. Implemented coils: coil A (left) and coil B (right) 
As can be seen in Fig. 3, critical current of both coils is similar and approximately $30 \mathrm{~A}$, which is only one third of the value given by manufacturer. Such value is coherent with results previously obtained by other research groups [10], and confirms that in $1 \mathrm{G}$ tapes the critical current value decreases rapidly with the increase of the magnetic field. It is also shown that when the two coils are operating in series, critical current value decreases and the losses on the tape increase. This also validates the fact that the increase of magnetic field decreases critical current value, since a set of two coils generate higher magnetic field than a single coil, for the same current.

\subsection{Improvement in Critical Current}

The degradation of critical current value measured in the SC coils is due to the existence of self field. As mentioned, this effect is accentuated for field components perpendicular to the tape surface, which is more relevant in the internal and external turns of the coils. One approach to minimize this effect consists on using ferromagnetic flux diverters on the extremities of the SC coil [11]. If the air gap between the SC coil and the ferromagnetic material is small enough then flux lines close through the ferromagnetic material and are diverted from the SC tape.

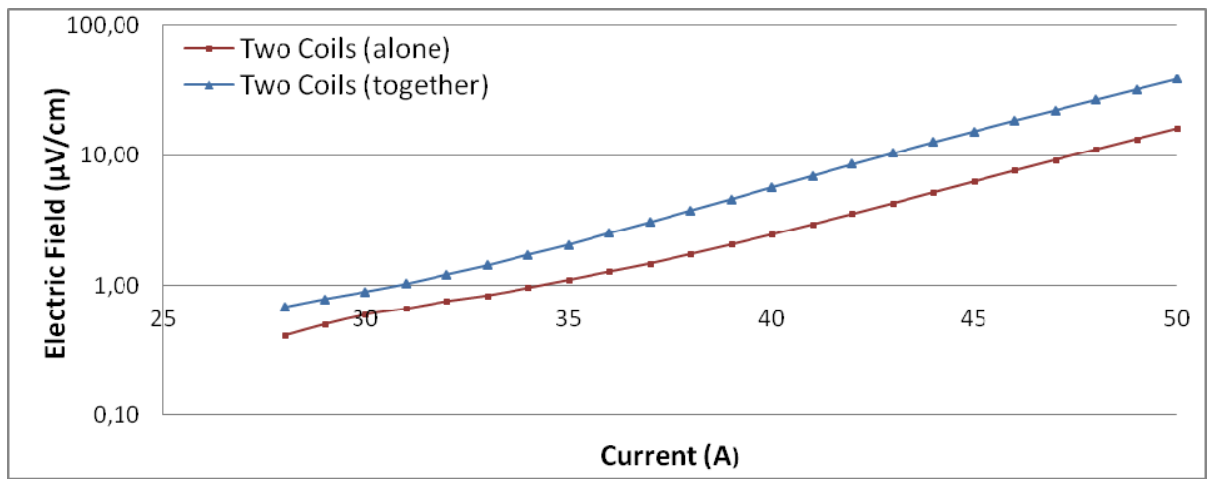

Fig. 3. Critical current measurement

To verify the previous concept, the SC coil built by two series connected pancakes, was tested again with the ferromagnetic material on top and beneath. Fig. 4 shows a comparison between the results obtained with and without diverters. According to that figure, critical current increased with diverters, which is accomplished by a decrease in resistive losses in the SC tape. This demonstrates that ferromagnetic diverters have a positive effect in the field distribution. 


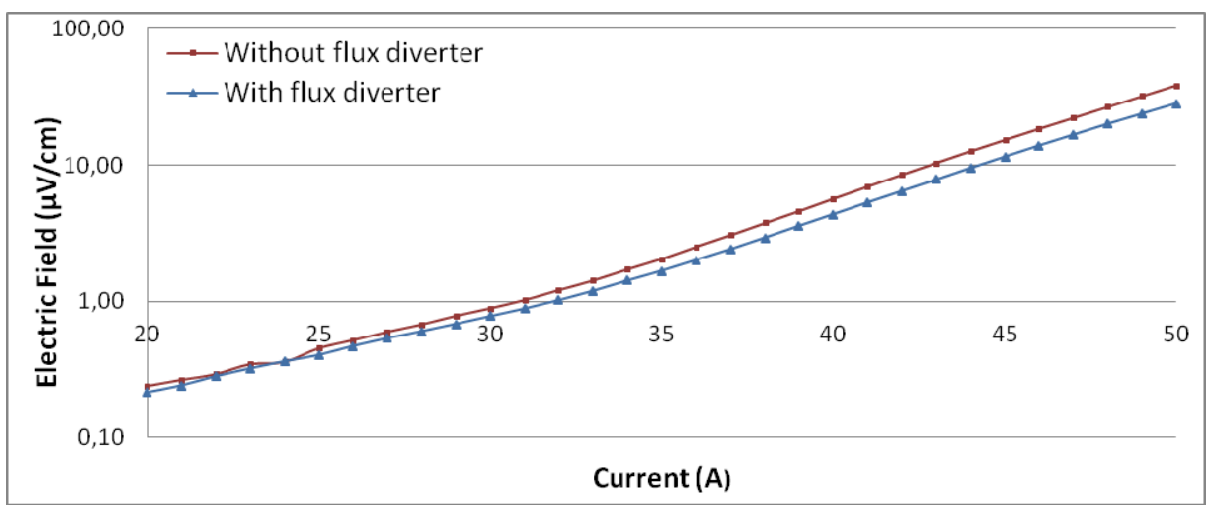

Fig. 4. Critical current measurement with flux diverter

\section{Conclusions}

In the design of a SMES system, several steps must be followed. The superconducting coil must be properly modeled as it represents the core element of the device. As a first approach, a mathematical model to determine the inductance of superconducting coils was applied. This model is based on classical mathematical expressions, usually applied to conventional materials. To validate the model, two superconducting pancake coils were built using first generation tape and the inductance of the set was measured. The critical current of the set was measured and the influence of the magnetic field in the critical current value was verified. This is also very important on SMES because the stored energy depends on the squared value of current that flows through the coil. As a possible solution to minimize the effect of the magnetic field on the critical current value, a ferromagnetic diverter was added to the system leading to an increase in the current.

As future work, a complete model of the whole system, including mechanical and thermal effects is foreseen.

Acknowledgments. This work was supported by Fundação para a Ciência e a Tecnologia (Centre of Technology and Systems multiannual funding) under the framework of project PEst-OE/EEI/UI0066/2011.

\section{References}

1. Amaro, N., Murta Pina, J., Martins, J., Ceballos, J.M.: Superconducting Magnetic Energy Storage - A Technological Contribute to Smart Grid Concept Implementation. In: Proceedings of the 1st International Conference on Smart Grids and Green IT Systems, pp. 113-120. SciTePress - Science and Technology Publications (2012)

2. Eurelectric: Power Quality in European Electricity Supply Networks, Brussels (2003)

3. Benysek, G.: Improvement in the Quality of Delivery of Electrical Energy using Power Electronics Systems. Springer (2007) 
4. Matsushita, T., Himeda, Y., Kiuchi, M., Fujikami, J., Hayashi, K.: Characterization of Critical Current Density in Silver-Sheathed Bi-2223 Tape. IEEE Transactions on Applied Superconductivity 15, 2518-2521 (2005)

5. E.U., E. union: European SmartGrids Technology Platform - Vision and Strategy for Europe's Electricity Networks of the Future. Office for Official Publications of the European Communities, Brussels (2006)

6. Gungor, V.C., Sahin, D., Kocak, T., Ergut, S., Buccella, C., Cecati, C., Hancke, G.P.: Smart Grid Technologies: Communication Technologies and Standards. IEEE Transactions on Industrial Informatics 7, 529-539 (2011)

7. Rosa, E.B., Grover, F.W.: Formulas and Tables for the calculation of mutual and selfinductance. United States Government Printing Office, Washington (1948)

8. Babic, S., Salon, S., Akyel, C.: The Mutual Inductance of Two Thin Coaxial Disk Coils in Air. IEEE Transactions on Magnetics 40, 822-825 (2004)

9. Amaro, N., Murta Pina, J., Martins, J., Ceballos, J.M., Álvarez, A.: A fast algorithm for initial design of HTS coils for SMES applications. IEEE Transactions on Applied Superconductivity (preprint version) (2012), doi: 10.1109/TASC.2012.2231912

10. Jiang, Z.Q., Jin, J.X.: Critical current measurement and experimental comparison of $1 \mathrm{G}$ and 2 G HTS tapes. In: 2011 International Conference on Applied Superconductivity and Electromagnetic Devices, pp. 145-149 (2011)

11. Pardo, E., Šouc, J., Vojenčiak, M.: AC loss measurement and simulation of a coated conductor pancake coil with ferromagnetic parts. Superconductor Science and Technology 22, 075007 (2009) 